\title{
The Application of Factor Analysis in Construction Project Post-evaluation
}

\author{
Hai Yan Zhang ${ }^{1, \text { a }}$,Jin Wei Zhao ${ }^{2, b}$ \\ ${ }^{1}$ Building And Civil Engineering College Of Inner Mongolia University Of Science And \\ Technology,BaoTou City Inner Mongolia Autonomous Region, China \\ ${ }^{2}$ Mintal Group Co,Ltd, BaoTou City Inner Mongolia Autonomous Region, China \\ aZhanghaiyan197112@163.com, bztyzhy@126.com
}

\begin{abstract}
Keywords: construction project; post-evaluation; evaluation method; factor analysis
Abstract.Project after the authenticity of the evaluation results to a large extent depends on the rationality of the evaluation method, this article in view of the construction project post-evaluation of current method is a systematic research, and put forward the factor analysis method used in the construction project evaluation analysis, the use of dimension reduction multiple related indexes for the comprehensive factor, a few of them had nothing to do with each other to overcome the defects of the artificial weights are objective, authentic, only makes the comprehensive evaluation results.
\end{abstract}

\section{Introduction}

The implementation of construction project may involve many aspects of society, and therefore their success or failure depends on factors more aspects. These factors determine the success of the project must be determined through a comprehensive, objective, detailed analysis and evaluation will be. So late evaluation of the project is a very necessary part, through the evaluation can be informed of the specific circumstances of each factor to determine the success of the project. Ultimately lessons learned feedback to project decision-making, management and builders, for construction projects have been perfect, it is of great significance in improvements and construction projects to be built in the project decision-making.

In the process of post project evaluation, the selection of evaluation method is very important, whether the accurate and objective post evaluation results can be obtained, and the selection of evaluation methods plays a decisive role.

\section{Analysis and Comparison of Existing Methods of Evaluation}

At present, most of the methods used in project post evaluation are comprehensive evaluation. Commonly used comprehensive evaluation methods are: expert evaluation method; economic analysis method; operational research and other mathematical methods; intelligent evaluation methods.

Expert evaluation method currently has: scoring method, grading method, weighted scoring method and ordering, etc.. Compare to the other, the expert evaluation method is simple and practical, so it is widely used in many fields, but because of the subjective evaluation, the method relies on experts so often the emergence of the phenomenon of excessive proportion of subjective factors. Therefore, before choosing the evaluation method, we must carefully consider whether this method can be used.

Economic evaluation method is a good one as previously prepared comprehensive economic indicators to evaluate different objects methods. So far, the frequent uses of economic evaluation are: given a formula or method of calculation of the integrated model of economic indicators. The advantage of economic evaluation method is that the meaning is clear and unambiguous, so it can contribute to the comparative study of different objects. But the flaw is that the economic evaluation methods tend to apply to some specific formulas and models, which are often more difficult to complete, on the other hand, for those complex object systems, be given a uniform to a dimension ( for example, the growth rate) model is very difficult, thereby greatly limits the application of this method. 
Other operations research and mathematical methods: application of mathematical methods of operations research, etc., requires the user to have a more profound knowledge of mathematics, otherwise it is difficult to complete. Currently Mathematics project evaluation commonly used are: multi-objective decision-making method, data envelopment analysis methods and models (Charnes A, et a1 [1], 1978; Wei right age [2], 1988; Wei right age, Sun DB and Xiao Zhijie [3], 1991), AHP (analytic hierarchy process, AHP) (Saaty [4], 1980; Liu Bao et al [5], 1984), fuzzy comprehensive evaluation method, mathematical statistics.

DEA is designed for the relative effectiveness of multiple-input and multiple-output decision-making unit of a comprehensive evaluation. The method can be regarded as a non-parametric method of economic evaluation is based on a set of observations about the input-output to determine the effective production frontier of their nature. Wei Quanling by pareto DEA validity and effective multi-objective programming problem solution (non-dominated solutions) conducted a comparative study found that overall the two are equivalent. Data envelopment analysis method has been advances in technology assessment, and evaluation of a number of areas returns to scale enterprise efficiency evaluation is widely applied. DEA has applications in many other fields, such as enterprise performance evaluation (and Amendment Chuan and Chen Xiaohong [6], 2005).

The basic principle of AHP is based on having a hierarchical structure of goals, sub-goals (criteria), Constraints and departments of the object to evaluate and use pairwise comparison method of determining judgment matrix, then the biggest feature judgment matrix root corresponding eigenvector coefficients as respective components. Finally, a comprehensive program of each respective weight (priority).

Fuzzy comprehensive evaluation method is a method to target those systems with fuzzy comprehensive evaluation of factors. The fuzzy comprehensive evaluation method can better solve the comprehensive evaluation in fuzzy (such as things between generic not clarity, evaluation experts acknowledge the fuzziness, etc.) on the knowledge, so has been very widely used in many fields.

Finally, mathematical statistics used for project evaluation including principal component analysis, factor analysis, cluster analysis, discriminant analysis and the like. This method is widely used in many fields, including quality, economic benefits of environmental assessment, and time in the future, but also to more fields, is a very practical project evaluation methods.

\section{Characteristics and Advantage of Factor Analysis Method}

Factor analysis is one of the methods of mathematical statistics, is an objective method of empowerment. The use of dimension reduction, multiple related index in the comprehensive factor, for a handful of unrelated to each other and be able to give the meaning of each factor to clear, at the same time according to the size of each factor variance contribution rate to determine the weight value, overcomes the defect of human weights, objective, authentic, only makes the comprehensive evaluation results. But using this approach requires to have linear correlation between the initial number of observations.

The Characteristics of the Factor Analysis Method Factor analysis can be a number of observations of quantitative indicators for scientific classification. After evaluation of construction project, the evaluation index of the identified the initial index as observation index, the current the project evaluation are generally based on the experience of evaluators and classifies the knowledge of human to get it at the next higher level of qualitative indicators. According to the resulting qualitative indicators to determine the weights of every index, then to the evaluation results, will affect the reliability and objectivity of evaluation results. If use factor analysis to these observation index qualitative classification need to use mathematical method to calculate the calculated results, so that the results are more accurate.

Factor analysis method for each index weighting method is based on each index relative to the original variable information contribution to determine the size of. The more information you provide, the greater the empowerment. The eigenvalues of each common factor are lambda 1, lambda $=2, \ldots \mathrm{M}$, according to the contribution rate of eigenvalue, taking the proportion of the corresponding eigenvalue of each factor to the total extracted feature value as the weight. That is, 
the weight of each factor is(1):

$\lambda \mathrm{m} / \sum_{i=1}^{m} \lambda_{i}$

The Advantage of Factor Analysis Method. Construction project evaluation index is numerous, and most of the index and correlation between, therefore USES the factor analysis as a method for determining the weights of qualitative indicators, can simplify the analysis process, and can objectively give weight value, so it can ensure the reasonable accuracy of the evaluation results.

\section{Factor Analysis Application Cases}

Adopt the method of factor analysis to municipal heating co-generation project piping after comprehensive evaluation on the economic benefits after the operation of the project.

To Establish the Sample Observations. Selected indicators are time limit for a project schedule reasonable degree, investment payback period, the total investment yield, earnings before interest and tax, capital, Gold interest rates and financial net present value, financial, financial internal rate of return, net present value rate loan payback period, the asset-liability ratio.

And then with the X1, X2, X3, X4, X5, X6, X7, X8, X9 representation. Invited ten related senior experts, according to the various factors of scores, to percentile system, scoring the results are shown in table 1.

On sample observation value is obtained by kmo and Bartlett sphere test sample correlation matrixTo solve the characteristic equation of the correlation coefficient matrix $\mathrm{R}$, calculation for $\mathrm{R}$ $\mathrm{W}$ of characteristic vector and characteristic value of $\lambda$.

TABLE I. TABLE TyPe STYLES

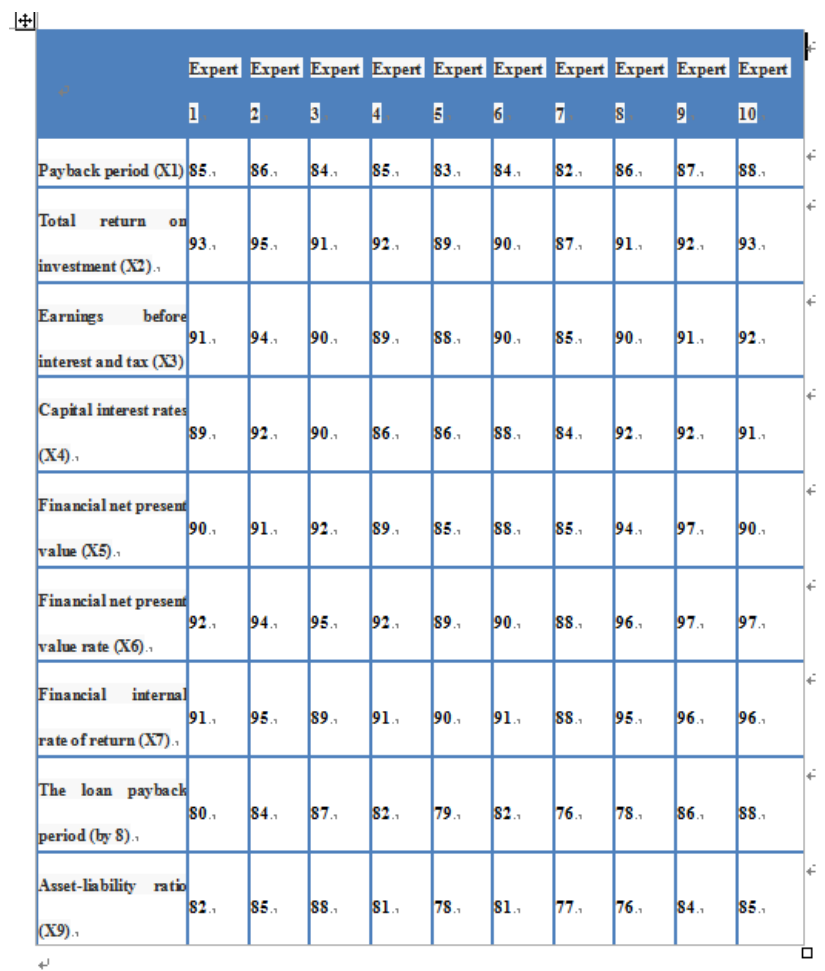


TABLE II. CORRELATION MATRIX

\begin{tabular}{|c|c|c|c|c|c|c|c|c|c|}
\hline & $\mathrm{x} 1$ & $\times 2$ & $x 3$ & $\mathrm{x} 4$ & $x 5$ & $x 6$ & $\pi 7$ & 88 & $x 9$ \\
\hline $\mathbf{x 1}$ & 1.00 & 6. 78 & b. 78 & b. 82 & b. 72 & b. 89 & p. 93 & b. 65 & 0.44 \\
\hline$x 2$ & b. 78 & 1.00 & b. 94 & b. 73 & b. 55 & 0.65 & b. 68 & b. 60 & 1. 62 \\
\hline x3 & 0. 78 & b. 94 & 1.00 & b. 84 & b. 58 & b. 69 & p. 74 & 6. 67 & 0.64 \\
\hline$\times 4$ & b. 82 & 0. 73 & b. 84 & 1.00 & b. 85 & 0. 89 & p. 83 & b. 59 & p. 49 \\
\hline$x 5$ & b. 72 & b. 55 & b. 58 & b. 85 & 1.00 & 0.88 & p. 69 & 6. 51 & 0.41 \\
\hline$x 6$ & 0.89 & b. 65 & 0.69 & 0. 89 & b. & 1.00 & p. 79 & 1. 70 & p. 54 \\
\hline $\mathbf{X 7}$ & b. 93 & b. 68 & b. 74 & b. 83 & 0.69 & 0. 79 & 1.00 & b. 46 & 0.22 \\
\hline$x 8$ & b. 65 & 0. 60 & b. 67 & b. 59 & b. 51 & b. 70 & P. 46 & 1.00 & b. 91 \\
\hline $\mathbf{x g}$ & 0.44 & 0.62 & 0.64 & 0.49 & 0.41 & 0. 54 & 1. 22 & 1. 91 & 1.00 \\
\hline
\end{tabular}

Determining Factor Number M. Results from table 3, according to the characteristic value is greater than 1, or carried out in accordance with the cumulative contribution rate of $85 \%$ for the principle factors. Current $\mathrm{k}$ factor accumulation of information the cumulative contribution rate exceeds $85 \%$, the $\mathrm{k}$ factor index instead of the original index $\mathrm{n}$. Through SPSS factor analysis of variance decomposition factor extraction conditions (see table 4), we can find that factor 1 , and factor variance contribution rates of 2 were $54.25 \%, 31.88 \%$ and total $86.1 \%>85 \%$. So number for the two factors.

TABLE III. Characteristic Value And Variance Contribution to the Table

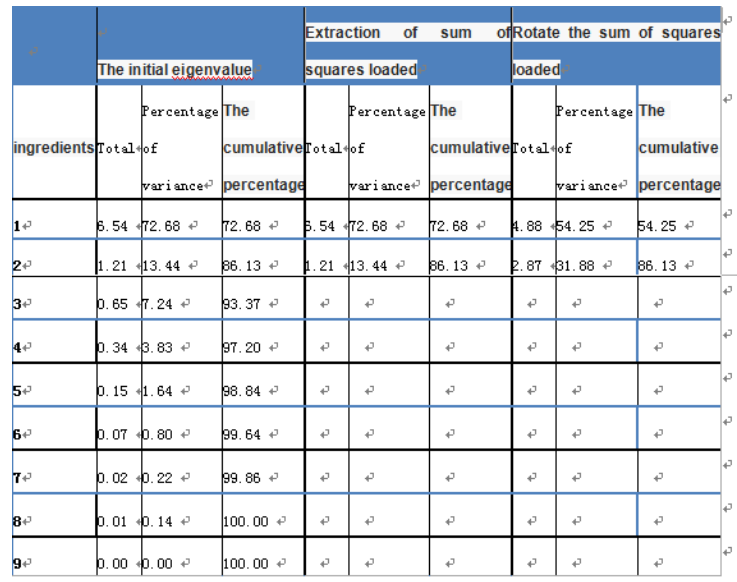

Factor Interpretation. Obtained from Figure 1 and table 4, factor1 in the original variables $\mathrm{x} 1, \mathrm{X} 2$, X3, x4, X5, X6, X7 have larger load, respectively, 0.89, 0.64, 0.69, 0.88, 0.82, 0.94. Reflects the financial profitability, can be named financial profitability. Factor 2 has large loads on the original variables X8 and x9, which are 0.88 and 0.98 , respectively. Reflects the solvency situation, it can be named Project solvency.

According to the implementation of the project economic benefit post evaluation of the two factors index weight shows, financial profitability for the maximum weight (Table 4) that project financial profitability are the main factors of the post evaluation of the economic benefits of the project. And then the ability to pay off the project.

\section{Factor Score Model}

$\bullet \mathrm{F} 1=0.038 \mathrm{X} 1+0.086 \mathrm{X} 2+0.022 \times 3-0.033 \mathrm{X} 4-0.056 \mathrm{X} 5+0.141 \mathrm{X} 6+0.138 \mathrm{X} 7+0.086 \mathrm{X} 8+0.068 \mathrm{X} 9$

$\bullet \mathrm{F} 2=0.022 \mathrm{X} 1+0.153 \mathrm{X} 2-0.029 \mathrm{X} 3-0.012 \mathrm{X} 4-0.093 \mathrm{X} 5+0.052 \mathrm{X} 6+0.025 \mathrm{X} 7+0.166 \mathrm{X} 8+0.018 \mathrm{X} 9$

- Comprehensive factor score model: $\mathrm{F}=(54.25 / 86.13) \mathrm{F} 1+(31.88 / 86.13) \mathrm{F} 2=0.63 \mathrm{~F} 1+0.37 \mathrm{~F} 2$

\section{Summary}

After the project evaluation, process and evaluation of complex content, many methods of evaluation, which is more appropriate, is the decisive factor to determine the true degree of evaluation results. In this paper, a systematic analysis of some of the commonly used evaluation methods, and compared to determine the factor based on the analysis of the post evaluation is more 
reasonable. However, because of the initial variables adopted by factor analysis, it is concluded that the subjective factors are inevitable in the quantitative analysis, and the objectivity of the evaluation results is inevitable. Therefore, how to avoid the subjective factors in factor analysis, or a direction in the future research.

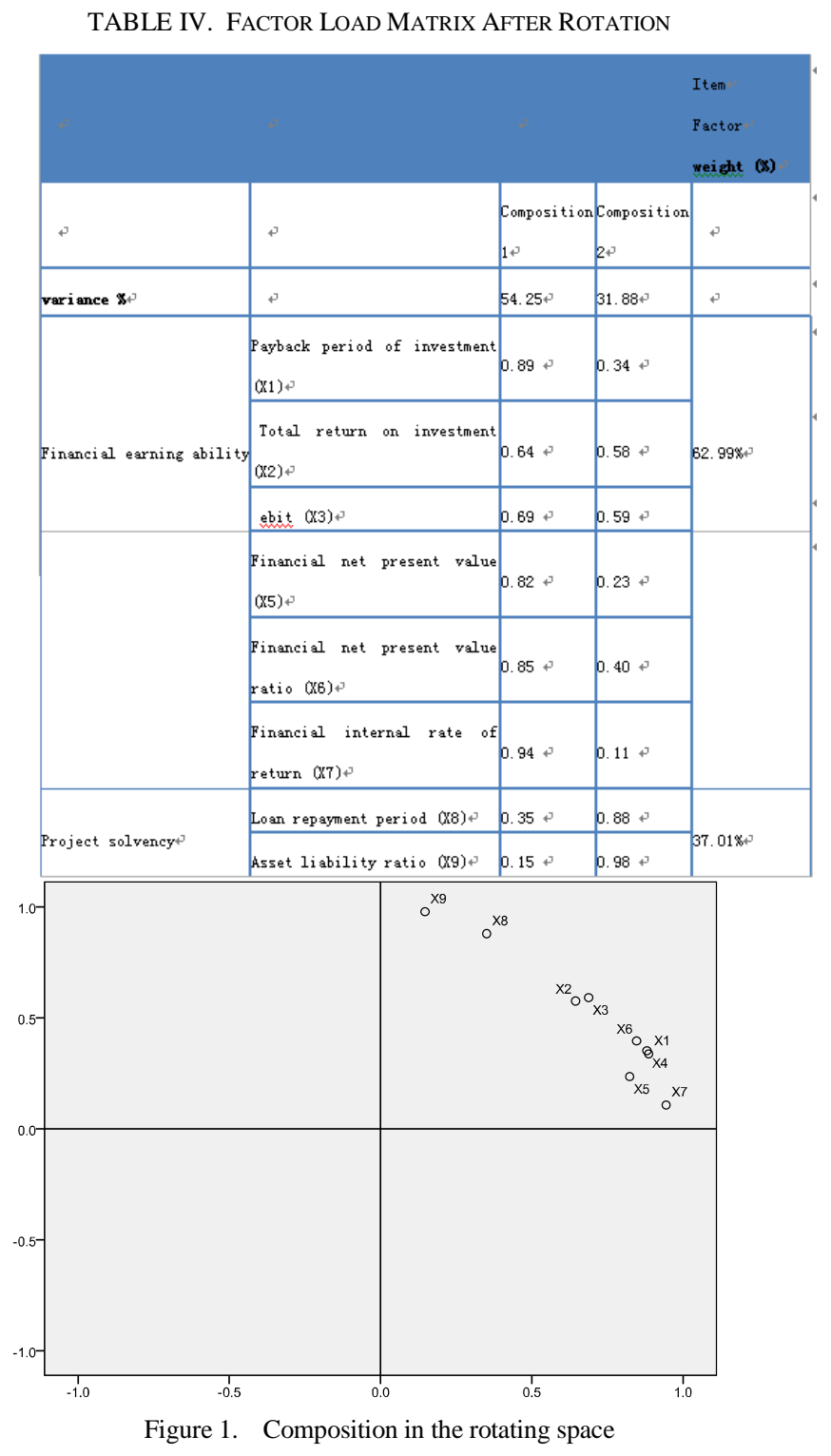

\section{References}

[1] Charnes A, et a1. Measuring the Efficiency of Decision Making Units. European Journal of Operations Research, 1978; 26(2): 429 444.

[2] Q.L. Wei. Evaluate the relative effectiveness of the DEA method. Beijing: Renmin University of China press, 1988.

[3] Q.L. Wei, D B Sun, Z.J. Xiao. DEA method and evaluation of technical progress, Chinese Journal of systems engineering, 1991.6 (2) 1 11.

[4] Saaty T.L. The Analytic Hierarchy Process, New York: MeGraw-HLL, 1980.

[5] B. Liu, Analytic hierarchy process (AHP) planning and decision making tools. System engineering, 1984; 2 (2): 23-30.

[6] S.Z. Chen, B.C. Lai, X.H. Chen.Evaluation of enterprise performance based on DEA method of system engineering, 2005, 23 (6) 99 100. 